\title{
Theoretical Formulation/Development of Signal Sampling with an Equal Arc Length Using the Frame Theorem
}

\section{Chunying Xu ( $\square$ chunyingxu@stu.edu.cn )}

Shantou University https://orcid.org/0000-0002-6298-1129

Junwei Hu

Zhejiang University

Jiawang Chen

Zhejiang University

Chen Cao

Zhejiang University

\section{Youngqiang Ge}

Zhejiang University

Miao Li

Charles Sturt University

\section{Research}

Keywords: Non-uniform spatial sampling, Equal arc length interval, Frame theorem, Reconstruction

Posted Date: November 17th, 2021

DOI: https://doi.org/10.21203/rs.3.rs-993947/v1

License: (c) (i) This work is licensed under a Creative Commons Attribution 4.0 International License.

Read Full License 


\title{
Theoretical Formulation/Development of Signal Sampling with an Equal Arc Length Using the Frame Theorem
}

\author{
Chunying $\mathrm{Xu}^{a, b, c, d^{*}}$, Junwei $\mathrm{Hu}^{d}$, Jiawang $\mathrm{Chen}^{d}$, Chen $\mathrm{Cao}^{d}$, Yongqiang $\mathrm{Ge}^{d}$, Miao $\mathrm{Li}^{e}$ \\ ${ }^{a}$ College of Engineering, Shantou University, 515013, China \\ ${ }^{b}$ Guangdong Provincial Key Laboratory of Digital Signal and Image Processing \\ ${ }^{c}$ Key Laboratory of Intelligent Manufacturing Technology (Shantou University), Ministry of
}

\section{Education}

${ }^{d}$ Ocean collage, Zhejiang University, Zhoushan, 316021, China

${ }^{e}$ Charles Sturt University, Albury, Australia

*Corresponding author. Chunying Xu

E-mail address: chunyingxu@ stu.edu.cn.

\begin{abstract}
Non-uniform sampling with equal arc length intervals can be found in shape measurements with contact sensor arrays. In this study, the conditions of non-uniform spatial sampling with an equal arc length interval are derived from two frame theorems. First, for general non-uniform sampling, the condition is that the equal arc length interval of the sensors should be less than $\frac{1}{4 \Omega}$. Second, for strictly increasing sampling, the condition is that the equal arc length interval of the sensors should be less than $\frac{1}{2 \Omega}$. The $\Omega$ is the maximum frequency of the detected object. For the latter,
\end{abstract}


if the sampling frequency is more twice than the sampling frequency required, the reconstruction error (RRMSE and MRE) is less than 5\%. If the sampling frequency is more than 2.5 times, the reconstruction error is less than $3 \%$. The simulation and the experiment are carried out and the results show that a sensor array with equal arc length interval can reconstruct the detected object with high accuracy.

Keywords: Non-uniform spatial sampling; Equal arc length interval; Frame theorem; Reconstruction.

\section{Introduction}

Sampling plays a critical role in signal analysis and contact sensor shape detection. However, samples cannot be collected uniformly in various applications, such as underwater terrain subsidence monitoring based on a Micro-ElectroMechanical System (MEMS) 9-axis sensor array[1-3], shape monitoring based on the Fiber Bragg Grating (FBG) sensor array[4-10], and data gloves or motion capture devices based on fiber curvature sensor arrays[11,12]. The sensor array has an implicit characteristic - the arc length interval between the sensors is equal and constant when the sensor array moves with the detected object. Therefore, the sampling of the sensor array becomes non-uniform even though the sensor array is deployed in an equal arc interval at the beginning (uniform sampling at the beginning) when the sensor array moves with the detected object.

Whether the detected object can be completely reconstructed from its nonuniform spatial sampled values with the condition of equal arc interval is a general 
and import problem which is should be studied. There are two reasons: one is that it cannot be sure if the reconstructed signal is not distorted from the non-uniform sampling set obtained from the contact sensor array. The other is that more sensors are often used to ensure the reconstruction without distortion, resulting in increased costs, more complex systems and larger data volumes if the non-uniform sampling conditions are not known.

Consequently, there have been many studies on randomized non-uniform sampling [13-18] and periodic non-uniform sampling [19-22]. For randomized nonuniform sampling, the non-uniform samples are often regarded as random perturbations from a uniform sampling grid in the analysis. The sampling times are often constructed by adding a Gaussian distribution sampling noise to the previous sampling time [23]. For periodic non-uniform sampling, the offset of sampling time $t_{n}$ from the equal interval sampling time sequence $n T$ has a periodic structure with a period of $M$, where $T$ is the average sampling period and $M$ is an integer. The sampling time is $t_{n}=n T+r_{n} T=(k M+m) T+r_{m} T$, where $r_{n} T$ is the offset of $t_{n}$ from $n T$, and $m \equiv n(\bmod M)$ [22]. This means that the adjacent sampling points are not uniform, but the interval between each sampling point and the subsequent sampling point is equal. The total sampling period is $M T$ [24,25]. It is often applied in analogto-digital (A/D) sampling technology, combining $\mathrm{M}$ AD converters with lower sampling rate to obtain one virtual sensor with high sampling rate [22]. There are also several algorithms in the literature that deal with the reconstruction of bandlimited signals from periodic non-uniform samples [19,26,27]. 
However, non-uniform sampling with the condition of equal arc interval is neither the randomized non-uniform sampling with a known distribution such as Gaussian distribution sampling noise nor periodic non-uniform sampling. There is no research on non-uniform spatial sampling for contact sensor arrays with the condition of equal arc interval. In this article, two theorems of non-uniform spatial sampling with the condition of equal arc interval are studied. The condition of non-uniform spatial sampling is derived from the frame theorem. For general non-uniform spatial sampling, the condition of sampling interval is obtained by the Kadec theorem. For the strictly increasing sampling sequence, the condition of sampling interval is obtained by the Benedetto theorem.

The rest of this paper is organized as follows. Section 2 introduces the conditions of non-uniform spatial sampling which are derived from the frame theorem. A simulation is presented in Sect. 3. An experiment is presented in Sect. 4 to evaluate the performance of the proposed sampling conditions. Finally, the conclusion is given in Sect. 5.

\section{Methods}

\subsection{Definition of non-uniform spatial sampling with the condition of equal arc}

\section{interval}

Fig. 1 and Fig. 2 show uniform and non-uniform spatial sampling respectively. The 'o' in Fig. 1 and Fig. 2 is the sampling point. In the uniform sampling, the interval of the $x$-axis is spaced equally, which is $x$ in Fig. 1. In the non-uniform case, 
the interval of the $x$-axis is spaced unequally which is $\left(x_{i}-x_{i-1}\right) \neq\left(x_{i+1}-x_{i}\right), i=0,1,2,3 \ldots$ (Fig 2) and the arc length of $\vec{f}\left(x_{i}\right) f\left(x_{j}\right), i \neq j$ is equal.

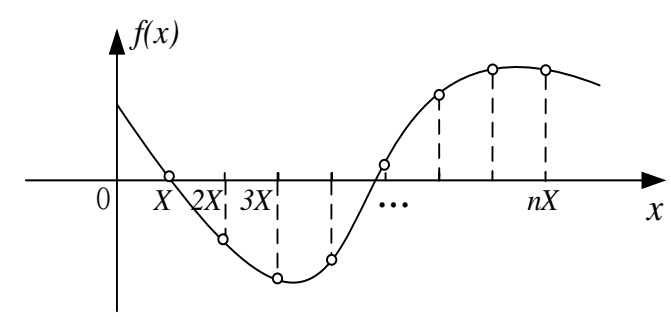

Fig. 1. Uniform spatial sampling.

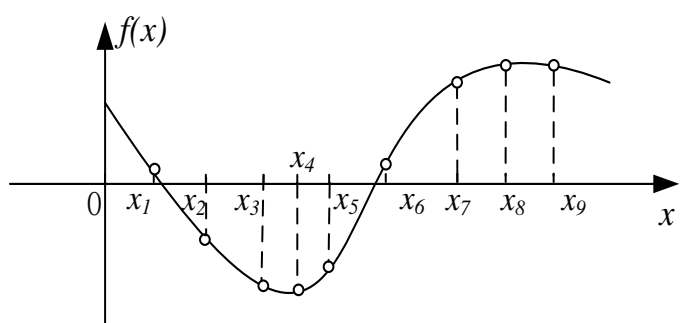

Fig. 2. Non-uniform spatial sampling.

\subsection{The mathematical expression of the non-uniform spatial sampling with the}

\section{condition of equal arc interval}

Assume that $f(x), x \in[0, L]$ is a mathematical expression of a detected object (such as a ribbon of terrain) which is a band limited function. The terrain is detected by a MEMS nine-axis sensor array [1-3]. In other words, $f(x)$ is sampled with the condition of equal arc length. $N$ is the sample number which can be obtained by $N=L / X$ ( $L$ is the arc length of the $f(x)$, and $x$ is the arc length of sensors which have an equal spatial interval at the beginning). Set $f_{s}(x)=f\left(x_{n}\right)$ is the sampling value. Like the process of uniform sampling, non-uniform sampling is the product of a series of impulse series and the original function. 

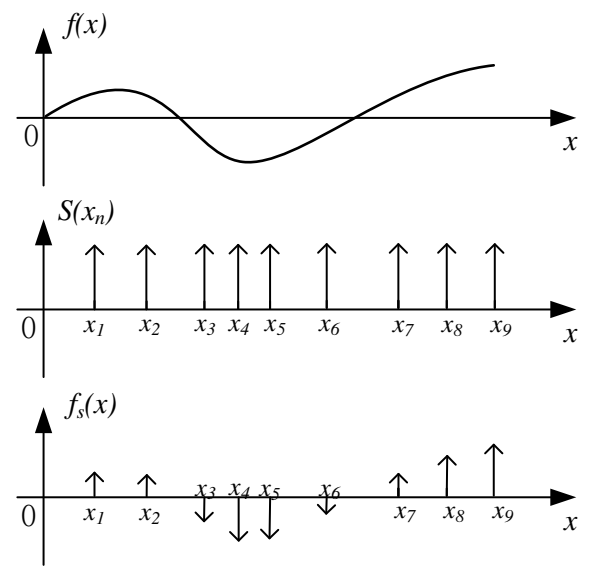

Fig. 3. The process of the non-uniform sampling.

Fig. 3 shows the process of non-uniform spatial sampling with equal arc length. In contrast to uniform sampling, the impulse series does not have periodicity (equal intervals), which can be expressed as:

$$
S\left(x_{n}\right)=\sum_{n=1}^{N} \delta\left(x-x_{n}\right)
$$

Where $x_{n}$ is the projection of the sampling point on the $x$-axis. Sampled signal $f_{s}(x)$ is the product of the impulse series $S\left(x_{n}\right)$ and the original function $f(x)$, which shows as:

$$
\begin{aligned}
f_{s}(x) & =f(x) \cdot S(x) \\
& =\sum_{n=1}^{N} f\left(x_{n}\right) \cdot \delta\left(x-x_{n}\right)
\end{aligned}
$$

As seen from formula (1), the sampled signal $f_{s}(x)$ is an impulse string weighted by the values $f(x)$.

\subsection{The condition of non-uniform sampling with equal arc length}

\subsubsection{Frame theorem}

A sequence $\left\{f_{n}, n \in \mathbf{Z}\right\}$ in a Hilbert space $\mathrm{H}$ is called a frame [23,27,28], if there 
exist constants $A, B>0$, such that for all $f \in H$,

$$
A\|f\|^{2} \leq \sum_{n \in \mathbf{Z}}\left|\left\langle f, f_{n}\right\rangle\right|^{2} \leq B\|f\|^{2}
$$

where \langle\rangle is inner product, which is defined as $\left\langle f, f_{n}\right\rangle=\int_{i} f(x) \overline{f_{n}(x)} d x\left(\overline{f_{n}(x)}\right.$ is the conjugation of $f_{n}(x),\|f\|$ is the norm of the $f$ which is $\|f\|=\langle f, f\rangle^{\frac{1}{2}}$.

In the frame theorem, the sampling point set is $\left\{x_{n}\right\}_{n \in \dot{\notin}}$ and sampling value set is $\left\{f\left(x_{n}\right)\right\}_{n \in \dot{\phi}}$. In order to completely reconstruct the band-limited function $f(x)$ from the sampling value set $\left\{f\left(x_{n}\right)\right\}_{n \in \dot{k}}$, the following inequality (4) should be met.

$$
A\|f\|^{2} \leq \sum_{n \in Z}\left|f\left(x_{n}\right)\right|^{2} \leq B\|f\|^{2}
$$

where $f(x) \in P W_{\Omega}=\left\{f(x) \in L^{2}(i), \operatorname{supp} \hat{f}(x) \subseteq[-\Omega, \Omega]\right\} . \Omega$ is the maximum frequency of function $f(x)$. When $\left\{e^{j 2 \pi \Omega x_{n}}\right\}_{n \in \dot{\phi}}$ is the frame for $P W_{\Omega}$, the function $f(x)$ can be reconstructed by the sampling value set $\left\{f\left(x_{n}\right)\right\}_{n \in \dot{\phi}}$.

\subsubsection{Condition of non-uniform sampling with equal arc length}

(1) The general non-uniform sampling with equal arc length

From the Kadec theorem[3], when the sampling point set $\left\{x_{n}\right\}_{n \in \dot{\phi}}$ satisfies inequality (5).

$$
\sup _{n \in Z}\left|x_{n}-\frac{n}{2 \Omega}\right|<\frac{1}{4} \times \frac{1}{2 \Omega}
$$

then $\left\{e^{j 2 \pi \Omega x_{n}}\right\}_{n \in \phi}$ is the frame of $P W_{\Omega}$. Inequality (6) can be expanded as:

$$
-\frac{1}{4} \times \frac{1}{2 \Omega}<x_{n}-\frac{n}{2 \Omega}<\frac{1}{4} \times \frac{1}{2 \Omega}
$$

Then inequality (7) can be expressed as:

$$
-\frac{1}{8 \Omega}+\frac{n}{2 \Omega}<x_{n}<\frac{1}{8 \Omega}+\frac{n}{2 \Omega}
$$

From inequality (7), it can be concluded that when the spatial arc length interval 
of the sensor array is not greater than $\frac{1}{4 \Omega}$, there is more than one sampling point in the range $\left[(n-1) \frac{1}{4 \Omega}, n \frac{1}{4 \Omega}\right], n=1,2, \ldots$, and the sampling point set is denoted as $\left\{x_{n}\right\}$. Then values can be extracted from the set $\left\{x_{n}\right\}$ and form a new set $\left\{f_{n}\right\}$ which should satisfy the inequation (7). The derivation process is as follows: Fig. 4 shows the derivation schematic diagram of the spatial sampling interval. Taking $n=1$ for example, assume that there is a sampling point in the range $\left[\frac{1}{4 \Omega}, \frac{1}{2 \Omega}\right]$. When the sampling point is in the range $\left[\frac{3}{8 \Omega}, \frac{1}{2 \Omega}\right]$ (dash area in the Fig. 4), inequality (7) is satisfied. When the sampling point is in the range $\left[\frac{1}{4 \Omega}, \frac{3}{8 \Omega}\right]$ (blue area in Fig. 4), then there must be another sampling point in the range $\left[\frac{3}{8 \Omega}, \frac{5}{8 \Omega}\right]$ (yellow area in Fig. 4), satisfying inequality (7). This is because the interval of the range $\left[\frac{3}{8 \Omega}, \frac{5}{8 \Omega}\right]$ and maximum spatial interval is equal, which is $\frac{1}{4 \Omega}$. Moreover, when there is more than one sampling point in the range $\left[\frac{1}{4 \Omega}, \frac{1}{2 \Omega}\right]$, the derivation above is also set up. Therefore, a new set $\left\{\ell_{n}, n=1,2, \ldots, N\right\}$ always can be extracted which satisfies inequality (7). In other words, when the arc length interval of the sensor array is not greater than $\frac{1}{4 \Omega}$, inequality (5) is satisfied. The frame bound A and B are not given in inequality (4). It is given by theorem 1 .

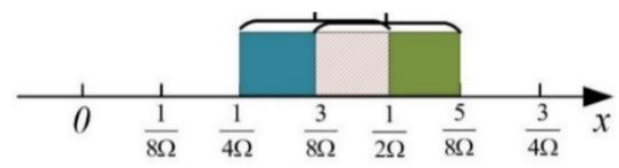

Fig. 4. Derivation schematic diagram of the spatial sampling interval.

Theorem 1: Assume that there are constant $\varepsilon, \alpha$ and $\beta, 0<\varepsilon<1, \alpha>0$, $\beta>0$, and sampling point set $\left\{x_{n}\right\}_{n \in \mathbb{k}}$ satisfied the following two inequations [3], denoted as condition 2 and condition 3 respectively: 


$$
\left\{\begin{array}{l}
\left|x_{n}-x_{m}\right| \geq \alpha>0, \quad n \neq m \\
\sup _{n \in Z}\left|x_{n}-\varepsilon \frac{n}{2 \Omega}\right| \leq \beta<\infty
\end{array}\right.
$$

Then there exist constants $A$ and $B, 0<A \leq B$, that are related to $\varepsilon, \alpha$ and $\beta$ such that inequality (4) for all $f \in P W_{\Omega}$.

The sampling point set $\left\{x_{n}\right\}_{n \in \dot{\phi}}$ satisfies condition $2,\left|x_{n}-x_{m}\right| \geq \alpha>0$, when the detected object is not vertical (actually, the case of vertical is similar to the horizontal case). In other words, the spatial sampling interval between sampling points always is greater than zero, and there exists $\alpha$, which satisfies condition $2,\left|x_{n}-x_{m}\right| \geq \alpha>0$.

Condition $3 \sup _{n \in Z}\left|x_{n}-\varepsilon \frac{n}{2 \Omega}\right| \leq \beta<\infty$ can be interpreted that all sampling points $x_{n}$ need to satisfy $-\beta \leq x_{n}-\varepsilon \frac{n}{2 \Omega} \leq \beta$, which can be transformed as:

$$
-\beta+\varepsilon \frac{n}{2 \Omega} \leq x_{n} \leq \beta+\varepsilon \frac{n}{2 \Omega}
$$

As the above analysis shows, there must exist a sampling point in the range $\left[(n-1) \frac{1}{4 \Omega}, n \frac{1}{4 \Omega}\right], n=1,2, \ldots, 2 N$, which means that $(n-1) \frac{1}{4 \Omega} \leq x_{n} \leq n \frac{1}{4 \Omega}$. Therefore, in order to satisfy inequality (9), inequality (10) should be satisfied:

$$
\left\{\begin{array}{c}
\frac{n}{4 \Omega} \leq \beta+\varepsilon \frac{n}{2 \Omega} \\
-\beta+\varepsilon \frac{n}{2 \Omega} \leq \frac{n-1}{4 \Omega}
\end{array}\right.
$$

Add the two inequalities in (10), then we get $\beta \geq \frac{1}{8 \Omega}$. Set $\beta=\frac{1}{4 \Omega}$ and use in the inequality $\frac{n}{4 \Omega} \leq \beta+\varepsilon \frac{n}{2 \Omega}$ and simplify, we get $\varepsilon \geq \frac{1}{4}-\frac{1}{n}$. What is more, $0<\varepsilon<1$, setting $\varepsilon=\frac{1}{4}(n>0)$ is satisfied. Therefore, the sampling point set satisfies condition 2 and condition 3 at the same time when $\beta=\frac{1}{4 \Omega}$ and $\varepsilon=\frac{1}{4}$.

It can be seen from the above analysis that when the equal arc length interval 
between the sampling points is half of the Nyquist sampling interval or one quarter of the period corresponding to the highest frequency of the detected object, the original function $f(x)$ can be reconstructed completely.

However, the sampling interval based on the non-uniform sampling theorem is larger. The more sampling points, the more sensors, the more complicated is the sensor network and the more cost. In applications such as underwater seabed monitoring based on sensors, the sampling points are a strictly increasing sequence. Therefore, non-uniform sampling theorem for increasing sampling is introduced which can obtain a less sampling interval.

(2) The non-uniform sampling of a strictly increasing sequence with equal arc length

In the frame theory, Marvasti [27] and Benedetto [28] state that, if the sample points $\left\{x_{n}, n \in \mathrm{i}\right\} \subseteq \mathrm{i}$ are a strictly increasing sequence for which

$$
\lim _{n \rightarrow \pm \infty} x_{n}= \pm \infty
$$

And

$$
0<d \leq \inf _{n}\left(x_{n+1}-x_{n}\right) \leq \sup _{n}\left(x_{n+1}-x_{n}\right)=T<\infty
$$

For a given $\Omega>0$, assume that $\Omega_{1}>\Omega$ satisfies the condition

$$
2 T \Omega_{1}<1
$$

Then $\left\{e^{j 2 \pi \Omega x_{n}}, n \in \varnothing\right\}$ is a frame of $P W_{\Omega}$. Frame boundaries $A$ and $B$ satisfy inequalities $A \geq \frac{(1-2 T \Omega)^{2}}{T}$ and $B \leq \frac{4}{\pi^{2} \Omega d^{2}}\left(e^{\pi \Omega d}-1\right)$. Where $P W_{\Omega}$ is the Paley-Wiener space which is defined as $P W_{\Omega}=\left\{f(x) \in L^{2}(i): \operatorname{supp} \hat{f} \subseteq[-\Omega, \Omega]\right\}$. It is a Hilbert space, considered as a closed subspace of $L^{2}(i)$ taken with the $L^{2}$-norm. The support 
condition, supp $\hat{f} \subseteq[-\Omega, \Omega]$, is described by saying that $f$ is $\Omega$-bandlimited.

If the detected object is not a vertical line, such as the slope of detected terrain is less than $90^{\circ}$, the sampling point set $\left\{x_{n}, n=1,2, \ldots, N\right\}$ satisfies the condition of strict increase for all $x_{n}$. In practical applications, the detected object is limited, which means $\lim _{n \rightarrow \pm \infty} x_{n}<\infty$. The sampling point set and sampling values should be extended to satisfy the condition. The idea of extension is that for sampling points, the sampling points should strictly increase and the difference between adjacent sampling points is less than $\frac{1}{2 \Omega}$. Therefore, the extension sampling point set $\left\{\ell_{n}, n=-\infty, \ldots, 1,2, \ldots, \infty\right\}$ meets the condition of $\sup \left(\varnothing_{n+1}-\varnothing_{n}\right)<\frac{1}{2 \Omega}<\infty, 0<\inf \left(\varnothing_{n+1}-\varnothing_{n}\right)$ and $\lim _{n \rightarrow \pm \infty} \varnothing_{n}= \pm \infty$. For the sampling values, the extended sample values are set as zeros and the original sample values were kept unchanged. Denote the extension sampling value set as $\left\{f\left(\varnothing_{n}\right), n=-\infty, \ldots, 1,2, \ldots, \infty\right\} \quad, \quad f\left(\ell_{n}\right)=0 \quad$ was set when $n<1$ and $n>N$. $f\left(f_{n}\right)=f\left(x_{n}\right)$ was set when $n=1,2, \ldots, N$.

From the above derivation, it can be obtained that the non-uniform sampling point set and sampling values can completely reconstruct the original signal when the arc length interval of a sensor array is less than $\frac{1}{2 \Omega}$, and there should be more than one sampling point in the range $\left[(n-1) \frac{1}{2 \Omega}, n \frac{1}{2 \Omega}\right]=\left[(n-1) \frac{\pi}{3}, n \frac{\pi}{3}\right], n=1,2, \ldots, N$.

\section{Simulation}

\subsection{Simulation of general non-uniform spatial sampling theorem}

A simulation for the non-uniform sampling condition and reconstruction method is carried out. Any signal can be superimposed by sinusoidal signals and cosine signals. Therefore, a cosine signal and a sinusoidal complex signal are selected for the 
simulation. The cosine signal is $f(x)=\cos (3 x), x \in[0,10]$, and the sinusoidal complex signal is $f(x)=0.5 \sin (2 x)+\cos (3 x), x \in[0,10]$.

The maximum frequency of both signals is $\Omega=\frac{3}{2 \pi}$. According to the above analysis, the spatial sampling interval should be less than $\frac{1}{4 \Omega}=\frac{\pi}{6} \approx 0.52$. The sampling interval is regarded as 0.5 , and there must be more than one sampling point in the range $\left[(n-1) \frac{1}{4 \Omega}, n \frac{1}{4 \Omega}\right]=\left[(n-1) \frac{\pi}{6}, n \frac{\pi}{6}\right], n=1,2, \ldots, N$. As mentioned above, one sampling point in the range $\left[(n-1) \frac{1}{4 \Omega}, n \frac{1}{4 \Omega}\right]=\left[(n-1) \frac{\pi}{6}, n \frac{\pi}{6}\right], n=1,2, \ldots, N$ is the boundary condition (the sampling point set can reconstruct the original function completely in the worst case). Then the sampling number $N$ is $N=L / T=10 /(\pi / 6) \approx 19$. Fig. 5 and Fig. 6 are the cosine signal, sinusoidal complex signal, sampling points with random and equal arc length, reconstructing signals respectively. The random sampling points are random numbers in the range $\left[(n-1) \frac{1}{4 \Omega}, n \frac{1}{4 \Omega}\right]=\left[(n-1) \frac{\pi}{6}, n \frac{\pi}{6}\right], n=1,2, \ldots, N$ which are blue hollow circles in Fig. 5 and Fig. 6. The arc length between adjacent sampling points is not equal. The respective reconstructing signals are green dotted lines in Fig. 5 and Fig. 6. If the sampling points satisfy the condition with equal arc length, the interval of sampling points with equal arc length is $\frac{1}{4 \Omega}=\frac{\pi}{6}$ which are pink solid circles. The arc length interval is regarded as 0.5 . For the cosine signal, the arc length is 22.28 , the sampling number required is 46 . For the sinusoidal complex signal, the arc length is 21.81 , the sampling number required is 45 . The respective reconstructing signals are red dotted lines in Fig. 5 and Fig. 6. The solid black line in both figures is the original signal.

The reconstructing signals are basically consistent with the original signals. In 
order to evaluate the accuracy of the reconstruction, the relative root mean square error (RRMSE) and mean relative error (MRE) between the original signal and the reconstructing signal are calculated.

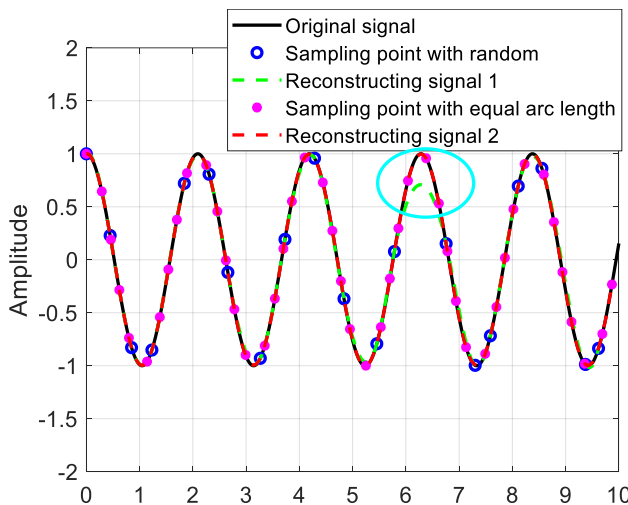

Fig. 5. Cosine signal, sampling points with random and equal arc length, and reconstructing signals respectively.

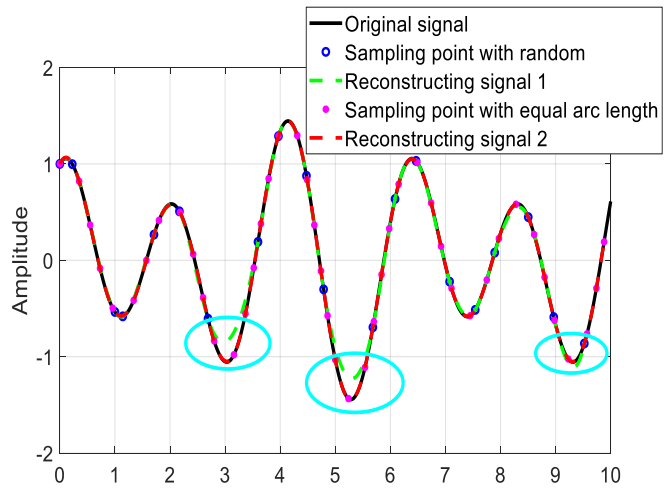

Fig. 5. Sinusoidal complex signal, sampling points with random and equal arc length, and reconstructing signals respectively.

From Table 1, Fig. 5 and Fig. 6, first, the reconstructing error of both is mainly at the extremum points (cyan areas in Fig. 5 and Fig. 6), where the non-uniform sampling points are far away from the extremum points. Therefore, the reconstructing error is related to the distribution of non-uniform sampling points. Second, the sampling numbers with equal arc length are more than random sampling, and the reconstructing error of sampling with equal arc length is much less than random sampling. This is because the arc length (about 22) is much longer than the $x$-axis range (from 0 to 10). The sampling number with equal arc length is more than twice the random sampling. According to the general non-uniform sampling theorem, there must be one sampling point in the range $[(n-1) * 0.5, n * 0.5], n=1,2, \ldots, 20$. And there are more than two sampling points in the range 
$[(n-1) * 0.5, n * 0.5], n=1,2, \ldots, 20 \frac{n !}{r !(n-r) !}$ when the arc length interval is 0.5. In other words, the sampling frequency is more than twice the sampling frequency of the general non-uniform sampling theorem. Moreover, when the signal is a line, the sampling points are uniform, the signal can also be reconstructed completely.

Table 1. The reconstructing error of non-uniform sampling with random and equal arc length.

\begin{tabular}{ccccccc}
\hline & \multicolumn{3}{c}{ Random sampling } & \multicolumn{3}{c}{ Sampling with equal arc length } \\
\hline & RRMSE & MRE & Sampling & RRMSE & MRE & Sampling \\
& $(\%)$ & $(\%)$ & number & $(\%)$ & $(\%)$ & number \\
\hline Cosine signal & 10.28 & 10.44 & 20 & 0.33 & 0.17 & 46 \\
\hline $\begin{array}{c}\text { Sinusoidal } \\
\text { complex signal }\end{array}$ & 10.23 & 13.22 & 20 & 0.34 & 0.21 & 45 \\
\hline
\end{tabular}

\subsection{Simulation of strictly increasing non-uniform sampling theorem}

The same simulation signals are selected. As mentioned above, there must be more than one sampling point in the range $\left[(n-1) \frac{1}{2 \Omega}, n \frac{1}{2 \Omega}\right]=\left[(n-1) \frac{\pi}{3}, n \frac{\pi}{3}\right], n=1,2, \ldots, N$ when the signal can be reconstructed completely, the number of sampling points should be more than $N=L / T=10 /(\pi / 3) \approx 10$ according to the strictly increasing non-uniform sampling theorem. In the practical application, the equal arc length is deployed on the arc, the spatial equal arc length interval should be less than $\frac{1}{2 \Omega}=\frac{\pi}{3} \approx 1.05$. Take the interval as 1 for example, the arc length of the cosine signal is 22.28 , and the arc length of the sinusoidal signal is 21.81 . The number of the sampling points is 23 . The sampling interval is shorter, sampling points are more, and the reconstruction accuracy is higher. The different sampling intervals are tested which progressively decrease from 1.02 to 
0.82 at an interval of $0.05(0.05 * 1.02)$. Fig. 7 shows the cosine signal, the sampling points with different arc length interval, reconstructing signals respectively, and Fig. 8 shows the ones of the sinusoidal complex signal. Table 3 shows the reconstruction error of non-uniform sampling with different equal arc length interval. The RRMSE and MRE between the original signal and the reconstructing signal are also calculated.

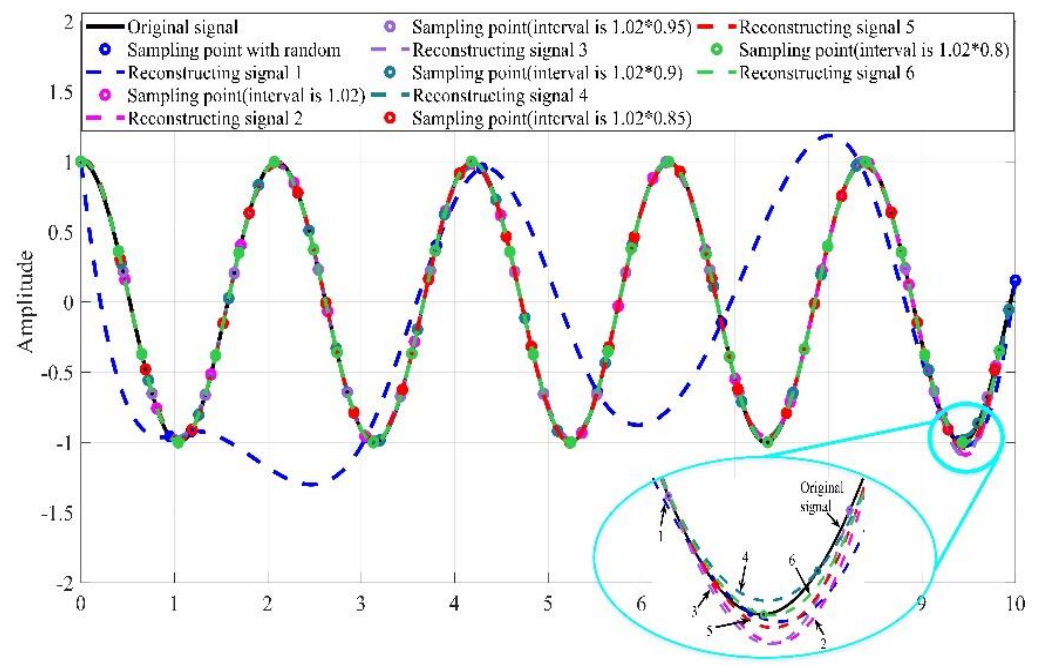

Fig. 7. The cosine signal, random sampling points, sampling points with equal arc length (interval is $1.02,1.02 * .095,1.02 * 0.90,1.02 * 0.85,1.02 * 0.80$ ) and reconstructing signals respectively.

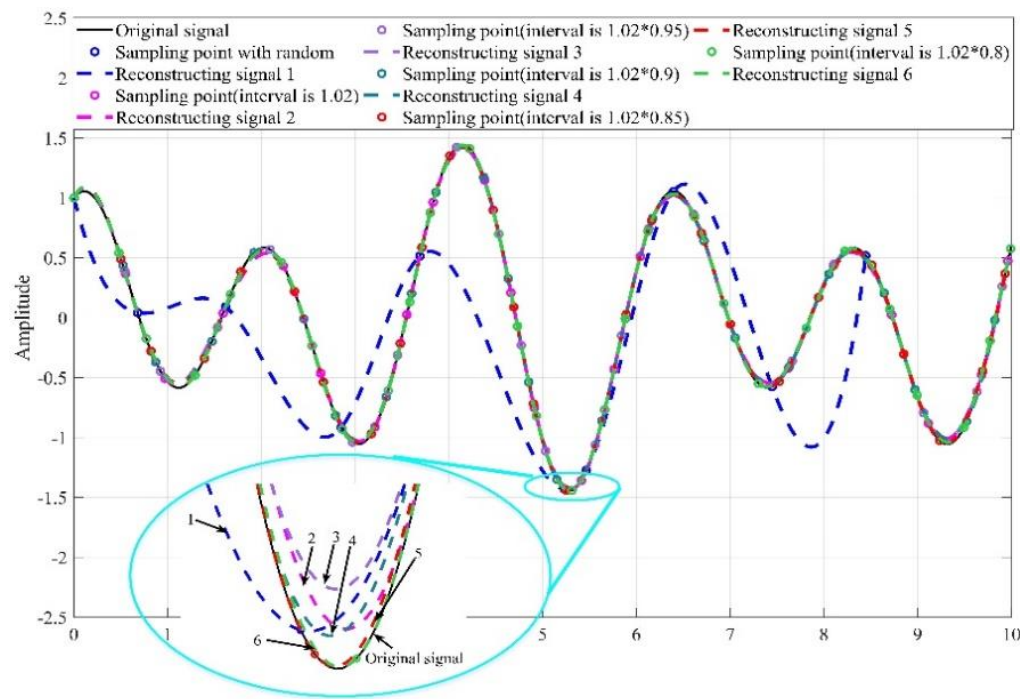

Fig. 8. The sinusoidal complex signal, random sampling points, sampling points with equal arc length (interval is $1.02,1.02 * .095,1.02 * 0.90,1.02 * 0.85,1.02 * 0.80$ ) and reconstructing signals 
respectively.

Table 2. The reconstruction error of non-uniform sampling.

\begin{tabular}{ccccccc}
\hline & \multicolumn{3}{c}{ Cosine signal } & \multicolumn{3}{c}{ Sinusoidal complex signal } \\
\hline $\begin{array}{c}\text { Arc length } \\
\text { interval }\end{array}$ & $\begin{array}{c}\text { RRMSE } \\
(\%)\end{array}$ & $\begin{array}{c}\text { MRE } \\
(\%)\end{array}$ & $\begin{array}{c}\text { Sampling } \\
\text { number }\end{array}$ & RRMSE (\%) & MRE (\%) & $\begin{array}{c}\text { Sampling } \\
\text { number }\end{array}$ \\
\hline $\begin{array}{c}1.02 \\
\text { (random) }\end{array}$ & 124.22 & 258.56 & 10 & 112.50 & 242.52 & 10 \\
\hline 1.02 & 4.04 & 2.31 & 23 & 3.81 & 4.85 & 23 \\
\hline 0.97 & 3.35 & 2.26 & 24 & 3.17 & 4.74 & 24 \\
$(0.95 * 1.02)$ & & & & & & \\
\hline 0.92 & 2.49 & 1.90 & 26 & 2.87 & 3.62 & 25 \\
$(0.90 * 1.02)$ & & & & & & \\
\hline 0.87 & 2.45 & 1.70 & 27 & 2.15 & 2.99 & 27 \\
$(0.85 * 1.02)$ & & & & & & \\
\hline 0.82 & 1.42 & 0.84 & 27 & 1.63 & 1.88 & \\
$(0.80 * 1.02)$ & & & & & & \\
\hline
\end{tabular}

From Fig. 7 Fig. 8 and Table 2, first, it can be seen that the arc length of the sinusoidal signal and sinusoidal complex signal is about the same, the same highest frequency, the same sampling points are needed when the arc length interval is the same. Second, the smaller the arc length interval, the more sensors are needed, and the less the reconstruction error. Three, the RRMSE and MRE of the reconstruction error are less than $5 \%$ when the arc length interval is 1.02 (the condition of the strictly increasing non-uniform sampling theorem). Those are less than $3 \%$ when the arc length interval is $0.87\left(0.85^{*} 1.02\right)$. This is because the condition of the strictly increasing non-uniform sampling theorem is that there must have more than one sampling point in the range $[(n-1) * 1.05, n * 1.05], n=1,2, \ldots, 10$. When the arc length interval is 1.02 , the number of sampling points is 23 and there are more than two 
sampling points in the range $[(n-1) * 1.05, n * 1.05], n=1,2, \ldots, 10$. In other words, the sampling frequency is more twice than the sampling frequency of the strictly increasing non-uniform sampling theorem. When the arc length interval is 0.87 $\left(0.85^{*} 1.02\right)$, the number of sampling points is 27 and the sampling frequency is more than 2.5 times the sampling frequency of the strictly increasing non-uniform sampling theorem. Fourth, compared to simulation of the general non-uniform spatial sampling (Table 1), the RRMSE and MRE is much bigger when the arc length interval is 1.02 which satisfies the condition of the strictly increasing non-uniform sampling theorem. This is because the signal in spatial domain is limited. The frequency spectrum is infinite. There is some energy missing when the highest frequency is regarded as $\Omega=\frac{3}{2 \pi}$. The arc length interval in the simulation of the general non-uniform spatial sampling is much less than the one in this case, and the accuracy is much higher. Moreover, when the signal is a line, the sampling points are uniform, the signal can also be reconstructed completely.

\section{Experiment and result discussion}

\subsection{Experimental setup}

The experimental setup mainly includes terrain deformation simulation platform, three-dimensional (3D) laser scanner, sensor array and computer for data processing and terrain display. The experimental setup is shown in Fig. 9. The deformation simulation platform is $2.1 \mathrm{~m} \times 1.8 \mathrm{~m}$ (the length along the sensor array is $2.1 \mathrm{~m}$, and the length in the direction perpendicular to the sensor array is $1.8 \mathrm{~m})$. Sixteen $(4 \times 4)$ screw slides were placed in the area. The distances between the screw slides were 70 
$\mathrm{cm}$ and $45 \mathrm{~cm}$. The heights of the screw slides are controlled by the motors. The shapes are constructed by the different heights of the screw slides. Six sensor arrays (Fig. 9) are arranged on the net and one end is fixed. Each sensor array is $2.1 \mathrm{~m}$ in length. The sensor array \#1, \#3, \#4 and \#6 is located directly above the screws, and their status can be changed directly by the screws. The shape change of sensor array has little effect on other sensor arrays. Therefore, the sampling of the sensor array is regarded as one-dimensional non-uniform sampling.

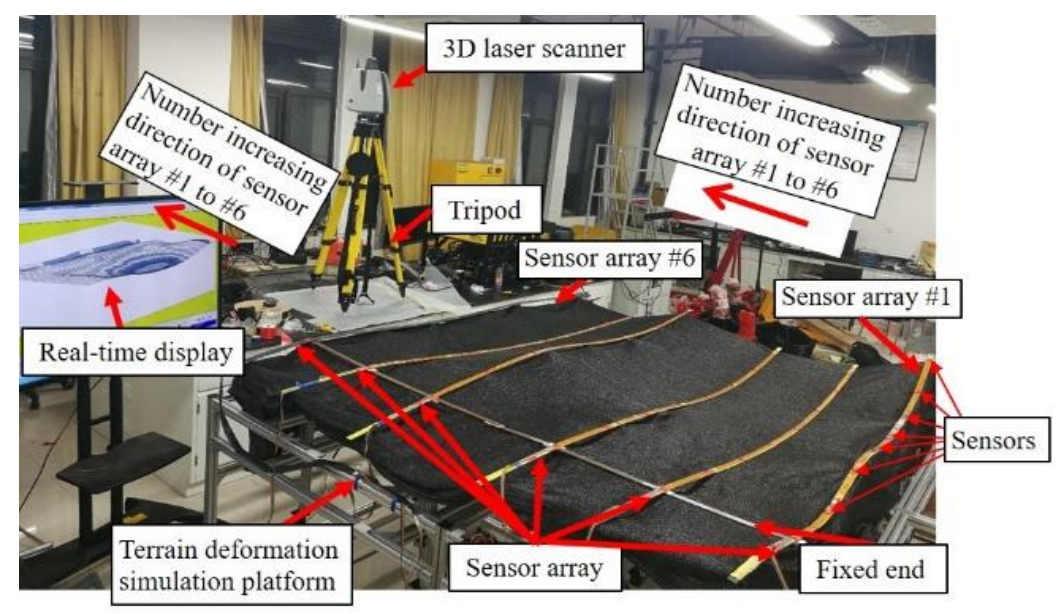

Fig. 9. The shape \#1 of simulation terrain.

The experimental setup includes a 3D laser scanner, and the data obtained by the $3 \mathrm{D}$ laser scanner will be used as the real value of the shape. The accuracy of the 3D laser scanner is $3 \mathrm{~mm}$. The $3 \mathrm{D}$ laser scanner scans the shapes, obtaining the point cloud data, which are processed by the software Cyclone (shown in Fig. 10), including point cloud data simplification, segmentation and coordinate extraction [32]. 


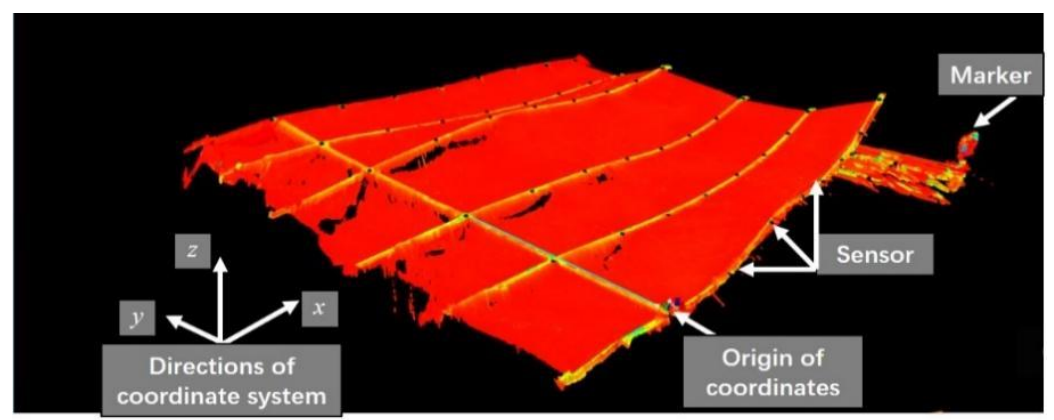

Fig. 10. The terrain data is obtained by $3 \mathrm{D}$ laser scanner.

In this study, we constructed three different shapes which are shown in Figs. 11 13. In the experiment of the terrain deformation monitoring, the sensors are placed with equal arc length. The samples are strictly increasing non-uniform. Therefore, we carried out the experiment for strictly increasing non-uniform sampling theorem.

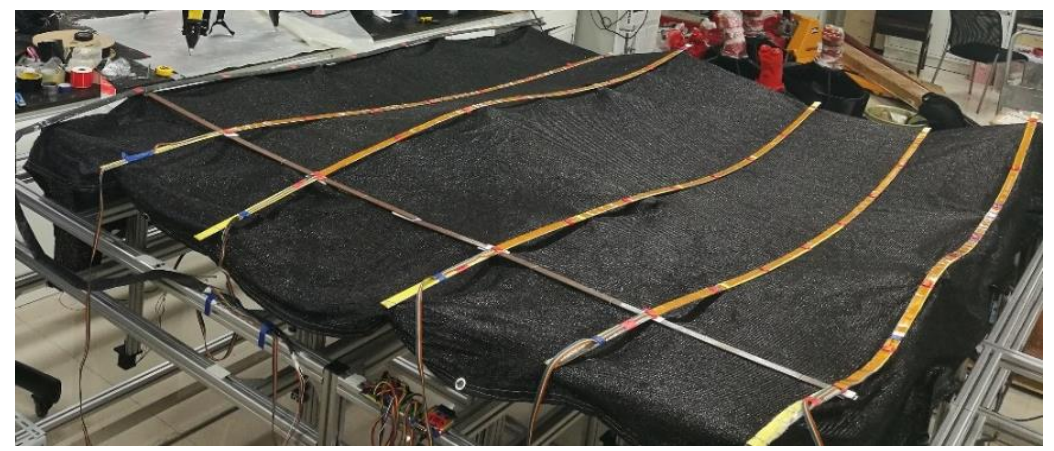

Fig. 11. The shape \#1.

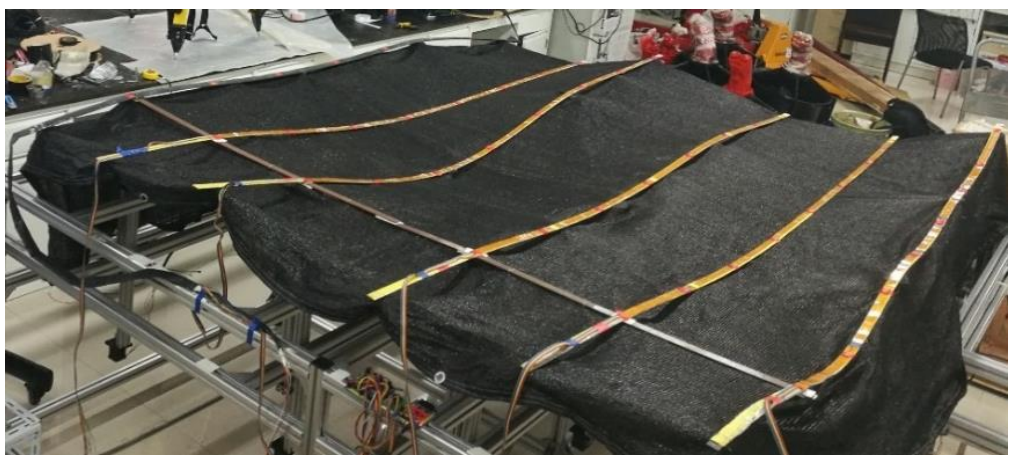

Fig. 12. The shape \#2. 


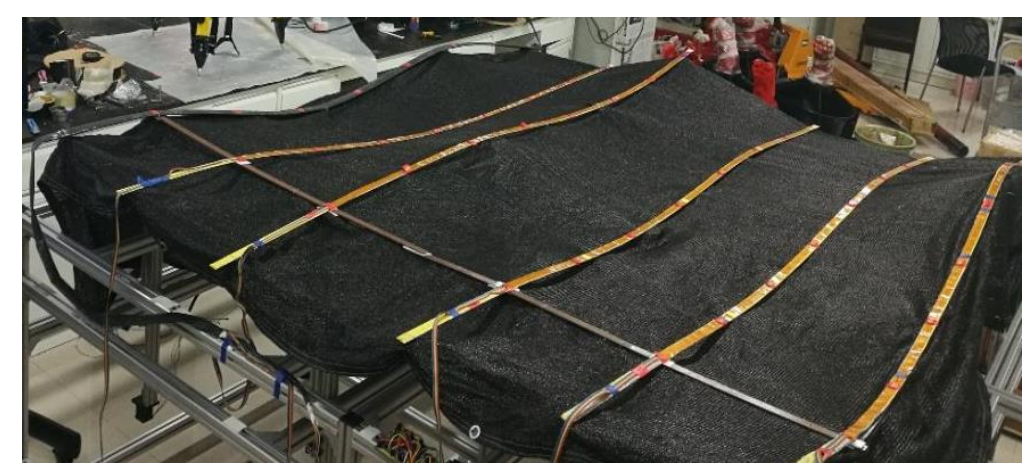

Fig. 13. The shape \#3.

\subsection{Results and discussion}

The one-dimensional amplitude spectrum of the shapes is calculated. The data are extracted at intervals of $0.02 \mathrm{~m}$ in the $x$-axis direction when $y=0.1 \mathrm{~m}, 0.2 \mathrm{~m} \ldots, 2.1 \mathrm{~m}$ (the interval is $0.3 \mathrm{~m}$ ). The sample number is 105 . The sampling frequency is $f_{s}=105$ $/ 2.1 \mathrm{~m}=50 \mathrm{~m}^{-1}$.

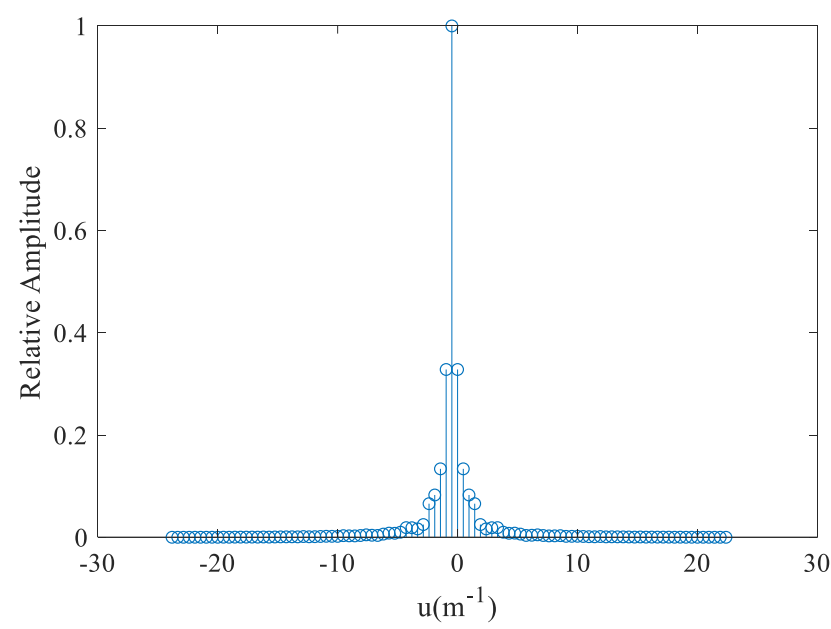

Fig. 14. The one-dimensional amplitude spectrum of terrain deformation shape \#1 (taking $\mathrm{y}=0 \mathrm{~m}$ as an example).

Fig. 14 shows the one-dimensional relative amplitude spectrum (the centralized spectrum) when the $y=0 \mathrm{~m}$. The terrain is limited in space, the frequency spectrum is infinite. The lowest frequency of the terrain is zero, and the highest frequency is 
defined as effective bandwidth in which the frequency range contains $95 \%$ energy of the shape. Table 3 to Table 5 are the highest frequency of these three shapes when the $y$ is fixed respectively. As can be seen from Fig. 14 and Table 3 to Table 5, the frequencies of the three shapes are all concentrated in the low frequency and the highest frequency $\Omega$ is $1.428 \mathrm{~m}^{-1}$. According to the non-uniform sampling theorem with equal arc length, the arc length interval between sensors should be less than $\frac{1}{2 \Omega} \approx 0.35 \mathrm{~m}$. The arc length interval is regarded as $0.3 \mathrm{~m}$ in this article.

Table 3. The highest frequency of shape \#1.

\begin{tabular}{ccccccccc}
\hline $\mathrm{y}(\mathrm{m})$ & 0 & 0.3 & 0.6 & 0.9 & 1.2 & 1.5 & 1.8 & 2.1 \\
\hline Highest frequency $\left(\mathrm{m}^{-1}\right)$ & 0.476 & 0.476 & 0.476 & 0.476 & 0.476 & 0.952 & 0.476 & 0.476
\end{tabular}

Table 4. The highest frequency of shape \#2.

\begin{tabular}{ccccccccc}
\hline $\mathrm{y}(\mathrm{m})$ & 0 & 0.3 & 0.6 & 0.9 & 1.2 & 1.5 & 1.8 & 2.1 \\
\hline Highest frequency $\left(\mathrm{m}^{-1}\right)$ & 0.952 & 0.476 & 0.476 & 0.952 & 1.428 & 0.952 & 0.476 & 0.476 \\
\hline
\end{tabular}

Table 5. The highest frequency of shape \#3.

\begin{tabular}{ccccccccc}
\hline $\mathrm{y}(\mathrm{m})$ & 0 & 0.3 & 0.6 & 0.9 & 1.2 & 1.5 & 1.8 & 2.1 \\
\hline Highest frequency $\left(\mathrm{m}^{-1}\right)$ & 0.476 & 0.476 & 0.476 & 0.476 & 0.952 & 0.476 & 0.476 & 0.952 \\
\hline
\end{tabular}

The sensor array senses the tilt angle of the shapes and conducts data collection, processing, modeling and display on a computer. The coordinates of the sensor array are used to analyse the reconstruction error. Table 6 shows the reconstruction error statistics. The RRMSE and MRE between the original signal and the reconstructing signal are also calculated.

Table 6. Reconstruction error statistics.

\begin{tabular}{llll}
\hline Shape & Sensor Array & RRMSE (\%) & MRE (\%) \\
\hline
\end{tabular}




\begin{tabular}{cccc}
\hline & Array \#1 & 6.57 & 6.57 \\
Shape \#1 & Array \#2 & 7.97 & 9.83 \\
& Array \#3 & 7.02 & 8.78 \\
& Array \#4 & 4.13 & 5.31 \\
& Array \#5 & 7.82 & 9.50 \\
& Array \#6 & 8.68 & 10.56 \\
\hline \multirow{3}{*}{ Shape \#2 } & Array \#1 & 5.04 & 6.31 \\
& Array \#2 & 8.68 & 10.73 \\
& Array \#3 & 7.12 & 8.66 \\
& Array \#4 & 4.97 & 5.58 \\
& Array \#5 & 8.84 & 10.00 \\
& Array \#6 & 5.98 & 7.18 \\
\hline Array \#1 & 4.26 & 6.44 \\
& Array \#2 & 8.77 & 10.42 \\
& Array \#3 & 7.19 & 9.71 \\
& Array \#4 & 3.03 & 5.21 \\
& Array \#5 & 7.87 & 9.37 \\
& Array \#6 & 3.09 & 4.57 \\
\hline
\end{tabular}

From Table 6, strong agreement is demonstrated between the data from the sensor array and data from the 3D laser scanner. The RRMSE is $8.84 \%$ (the sensor array \#5 of shape \#2). The maximum MRE is $10.56 \%$ (the sensor array \#5 of shape \#1). There are three main reasons for the errors. First, the measurement errors of sensors. According to the datasheet of the sensor, the angle error obtained by the MEMS accelerometer is $0.1^{\circ}$. The arc length interval is $30 \mathrm{~cm}$. The error cause by the sensor is $0.05 \mathrm{~cm}$, the MRE is $0.17 \%$. Second, the monitoring range of the terrain is limited. In other words, the signal is limited in the spatial domain. Though the arc length interval satisfies the strictly increasing non-uniform sampling theorem, the arc length interval accounts to reconstruction error. According to the above simulation, the error caused by the reconstruction error is less than 5\%. Three, the measurement errors may 
increase because the sensors could not fit well with the terrain surface when the terrain moves.

\section{Conclusion}

In this article, non-uniform spatial sampling with an equal arc length interval is presented. The definition of the non-uniform spatial sampling and associated mathematical expression is given. The condition of non-uniform spatial sampling is derived based on the frame theorem. Two simulations and an application (the terrain deformation test) of the strict increasing non-uniform sampling theorem are carried out. There are four conclusions:

(1) Though the condition of the strictly increasing non-uniform sampling theorem is satisfied, the RRMSE and MRE of the reconstruction signal is not close to zero. The reason is that the frequency spectrum is infinite when the signal in the spatial domain is limited, and there is some energy missing for the highest frequency which often is defined as effective bandwidth of signal. The effective bandwidth contains $95 \%$ of the energy of the signal.

(2) In practical application, sensors are deployed on the detected object. If the sampling point set is strictly increasing, the arc length interval between sensors can be based on the strictly increasing non-uniform sampling theorem, which $l<\frac{1}{2 \Omega}$. If the sampling frequency is more twice than the sampling frequency of the strictly increasing non-uniform sampling theorem, the reconstruction error (RRMSE and MRE) is less than 5\%. If the sampling frequency is more 2.5 times, the reconstruction error (RRMSE and MRE) is less than 3\%. If the sampling point set is not strictly 
increasing, the arc length interval between sensors should satisfy $l<\frac{1}{4 \Omega}$.

(3) Assume that the arc length of the detected object is $L$, the arc length interval of the sensor array is $l$. The sensor array with equal arc length can be deployed on which the highest frequency of the detected object is less than $\Omega<\frac{1}{4 l}$ or $\Omega<\frac{1}{2 l}$. For later, the $x$-axis coordinates of the detected object should be strict increasing.

(4) The smaller the arc length interval, the more sensors are needed, and the less the reconstruction error.

The results can guide technicians on the application of contact sensor array for shape monitoring with less sensors and high accuracy.

\section{Abbreviations}

RRMSE: relative root mean square error; MRE: mean relative error; MEMS: MicroElectro-Mechanical System; FBG: Fiber Bragg Grating; A/D: analog-to-digital; 3D: three-dimensional

\section{Authors' Contributions}

CYX, JWH, JWC, CC and YQG conceived and designed the experiments. CYX, CC and YQG performed the experiments. CYX analyzed the data. CYX and ML wrote the paper. All authors have contributed to this research work and read and approved the final manuscript.

\section{Funding}

This work was supported by National Natural Science Foundation of China (Grant No. 42106205 and 41976055), Guangdong Basic and Applied Basic Research Foundation 
(Grant No. 2019A1515110372 and 2021A1515011475), STU Scientific Research Foundation for Talents (Grant No. NTF19034).

\section{Availability of data and materials}

All data of simulation and experiment can be obtained by contacting the authors of the paper.

\section{Declarations}

\section{Ethics approval and consent to participate}

Not applicable.

\section{Consent for publication}

Not applicable.

\section{Competing interests}

The authors declare that they have no competing interests.

\section{References}

[1] C.Xu, J.Chen, H.Zhu, et al, Experimental Research on Seafloor Mapping and Vertical Deformation Monitoring for Gas Hydrate Zone Using Nine-Axis MEMS Sensor Tapes. IEEE J. Ocean. Eng. 44(4), 1090-1101(2018).

[2] C.Xu, J.Chen, H.Zhu, et al, Design and laboratory testing of a MEMS accelerometer array for subsidence monitoring. Rev. Sci. Instrum., 89(8), 085103 (2018). 
[3] C.Xu, J.Chen, H.Zhu, et al, Experimental Study on Seafloor Vertical Deformation Monitoring Based on MEMS Accelerometer Array. Paper presented at the 28th International Ocean and Polar Engineering Conference, Sapporo, 10-15 June 2018.

[4] T. Allsop,R. Bhamber, G. Lloyd, et al, Respiratory function monitoring using a real-time three-dimensional fiber-optic shaping sensing scheme based upon fiber Bragg gratings. J Biomed Opt.17(11), 17001(2012).

[5] M. Amanzadeh, S. Aminossadati, M. Kizil, et al. Recent developments in fibre optic shape sensing. Measurement 128 (2018): 119-137.

[6] Y. Du, B. Sun, J. Li, et al, Optical fiber sensing and structural health monitoring technology (Springer, Berlin Heidelberg New York, 2019).

[7] J. P. Moore and M. D. Rogge, Shape sensing using multi-core fiber optic cable and parametric curve solutions. Optics express 20(3), 2967-2973(2012).

[8] M. Abayazid, M. Kemp and S. Misra, 3D flexible needle steering in soft-tissue phantoms using Fiber Bragg Grating sensors. Paper presented at the 2013 IEEE International Conference on Robotics and Automation, Karlsruhe, 6-10 May 2013.

[9] R. J. Roesthuis, M. Kemp, J. J. Van Den Dobbelsteen, Three-dimensional needle shape reconstruction using an array of fiber bragg grating sensors. IEEE ASME Trans Mechatron19(4), 1115-1126(2013).

[10] S. C. Ryu and P. E. Dupont, FBG-based shape sensing tubes for continuum robots. Paper presented at the 2014 IEEE International Conference on Robotics and Automation, Hong Kong, 31 May-5 June 2014. 
[11] J. Chen, C. Cao, Y. Huang, Y. Zhang, et al, Research on Optical Fiber Sensor Based on Underwater Deformation Measurement. Sensors. 19(5), 1115(2019).

[12] C. Xu, K. Wan, J. Chen, et al, Underwater cable shape detection using ShapeTape. Paper presented at the OCEANS 2016 MTS/IEEE, Monterey, 19-23 September 2016.

[13] H. J. Landau, Necessary density conditions for sampling and interpolation of certain entire functions. Acta Mathematica. 117, 37-52(1967).

[14] H. J. Landau, Sampling, data transmission, and the Nyquist rate. Proc IEEE Inst Electr Electron Eng. 55(10), 1701-1706(1967).

[15] R. Venkataramani, Sub-Nyquist multicoset and MIMO sampling: Perfect reconstruction, performance analysis, and necessary density conditions, University of Illinois at Urbana-Champaign, 2001.

[16] S. Williams, Nonuniform random sampling: an alternative method of variance reductionfor forest surveys. Canadian journal of forest research. 31(12), 20802088(2001).

[17] L. Xu, F. Zhang, and R. Tao, Randomized nonuniform sampling and reconstruction in fractional Fourier domain. Signal Process. 120, 311-322(2016).

[18] K. Yao and J. B. Thomas. On some stability and interpolatory properties of nonuniform sampling expansions. IEEE trans. circuit theory 14(4), 404 -408(1967).

[19] H. Johansson and P. Löwenborg, Reconstruction of nonuniformly sampled bandlimited signals by means of digital fractional delay filters. IEEE Trans. Signal Process. 50(11), 2757-2767(2002). 
[20] D. G. Long and R. O. W. Franz, Band-limited signal reconstruction from irregular samples with variable apertures. IEEE Trans Geosci Remote Sens. 54(4), $2424-2436(2015)$.

[21] M. Rashidi and S. Mansouri, Parameter selection in periodic nonuniform sampling of multiband signals. Paper presented at the 2010 3rd International Symposium on Electrical and Electronics Engineering, Galati, 16-18 September 2010 [22] S. Zhao, R. Wang, Y. Deng, et al., Modifications on multichannel reconstruction algorithm for SAR processing based on periodic nonuniform sampling theory and nonuniform fast Fourier transform. IEEE J Sel Top Appl Earth Obs Remote Sens. 8(11), $4998-5006$ (2015).

[23] T. Strohmer and J. Tanner, Implementations of Shannon's sampling theorem, a time-frequency approach. Sampl Theory Signal Image Process. 4(1), 1-17(2005).

[24] S. Senay, Signal reconstruction from nonuniform samples using prolate spheroidal wave functions: theory and application, University of Pittsburgh, 2011.

[25] R. Tao, B. Li, Y. Wang, et al., On sampling of band-limited signals associated with the linear canonical transform. IEEE Trans. Signal Process. 56(11), 5454-5464 (2008).

[26] S. S. Goha and I. G. H. Ong, Reconstruction of bandlimited signals from irregular samples. Signal Process. 46(3), 315-329(1995).

[27] Farokh, M (ed.), Nonuniform sampling: theory and practice (Springer Science and Business Media, Berlin Heidelberg New York 2012). 
[28] J J. Benedetto, Irregular sampling and frames, wavelets: A Tutorial in Theory and Applications, 2, 445-507(1992).

[29] Y. C. Jenq, Digital spectra of nonuniformly sampled signals: Fundamentals and high-speed waveform digitizers. IEEE Trans Instrum Meas. 37(2), 245-251(1988).

[30] A. D. Poularikas (ed.), Transforms and applications handbook (CRC press, Boca Raton, 2018).

[31] S. Senay, Signal reconstruction from nonuniform samples using prolate spheroidal wave functions: theory and application, University of Pittsburgh, 2011.

[32] C.Xu, J.Hu, J.Chen, et al, Sensor Placement with Two-Dimensional Equal Arc Length Non-Uniform Sampling for Underwater Terrain Deformation Monitoring. J. Mar. Sci. Eng. 9(9), 954; https://doi.org/10.3390/jmse9090954 (2021). 


\section{Supplementary Files}

This is a list of supplementary files associated with this preprint. Click to download.

- declarationofcompetinginterests1.docx 